\title{
Review: screening instruments had sensitivities of $67-100 \%$ and specificities of $53-98 \%$ for detecting major depression in older primary care patients
}

Watson LC, Pignone MP. Screening accuracy for late-life depression in primary care: a systematic review. J Fam Pract 2003;52:95664.

Q What is the accuracy of various screening instruments for detecting depression in older adults in primary care?

\section{METHODS}

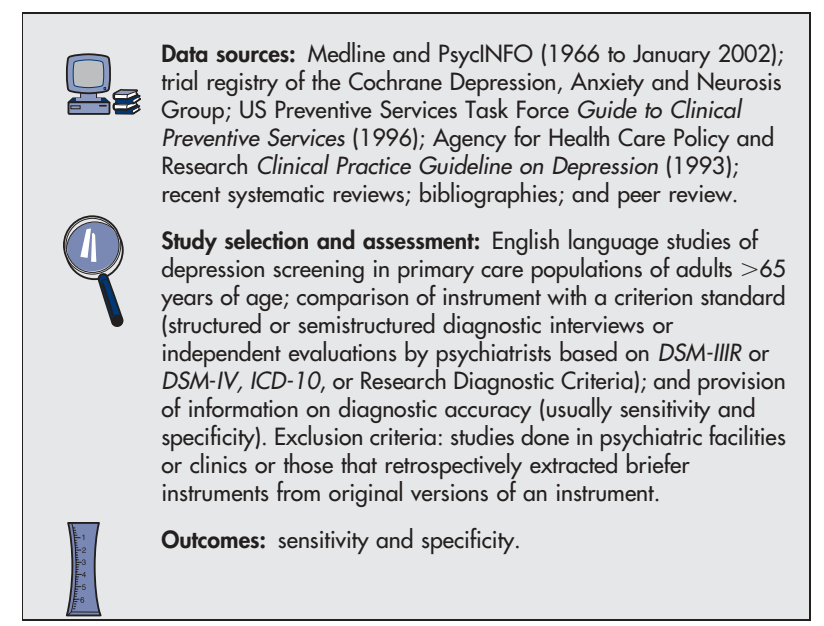

\section{MAIN RESULTS}

18 studies met the inclusion criteria. Meta-analysis was not possible because the studies included multiple screening instruments. 8 different instruments were assessed. The test characteristics of 7 instruments for detecting major depression are summarised in the table. Studies assessing detection of minor or subthreshold depression (Geriatric Depression Scale, Center for Epidemiologic Studies Depression scale, and General Health Questionnaire) reported sensitivities of $39-70 \%$ and specificities of $72-82 \%$.

For correspondence: Dr LCWats Medical Center, Durham, NC, USA. watso069@mc.duke.edu

Sources of funding: Robert Wood Johnson Clinical Scholars Program and Agency for Healthcare Research and Quality.

\section{CONCLUSION}

Overall, screening instruments had sensitivities of $67-100 \%$ and specificities of $53-98 \%$ for detecting major depression in older patients in primary care.

A modified version of this abstract appears in Evidence-Based Medicine.

Commentary

M any screening tools for clinical depression are now available for primary care practice. Watson and Pignone provide a useful and rigorous review of screening tools for older adults in primary care. Consistent with other reviews, the results show wide variations in sensitivity and specificity, ${ }^{1}$ which are indicators of screening tool accuracy. However, a given screening tool may perform better or worse depending on the population and setting, and even high quality screening tools are not necessarily accurate in populations with a low prevalence of the disorder. ${ }^{2}$ Screening tools may also lack accuracy for non-major depression, and overall, general screening programmes remain controversial. ${ }^{3}$ Detection of depression, although necessary, is not the only requirement for effective treatment to occur. ${ }^{4}$ Adequate treatment of depression in primary care may be impeded by various clinician, patient, structural, and process barriers, and these raise the question of the usefulness of simply implementing screening programmes. Such barriers to adequate treatment should constitute urgent agendas for both future interventions and health services research.

Celia E Wills, RN, PhD College of Nursing, Michigan State University East Lansing, Michigan, USA

1 Williams JW Jr, Pignone M, Ramirez G, et al. Identifying depression in primary care: a literature synthesis of case-finding instruments. Gen Hosp Psychiatry 2002;24:225-37.

2 Stommel M, Wills CE. Clinical research: concepts and principles for advanced practice nurses. Philadelphia: Lippincott Williams and Wilkins, 2004.

3 Pignone MP, Gaynes BN, Rushton JL, et al. Screening for depression in adults: a summary of the evidence for the U.S. Preventive Services Task Force. Ann Intern Med 2002;136:765-76.

4 Simon GE. Can depression be managed appropriately in primary care? J Clin Psychiatry 1998;59(Suppl 2):3-8.

Test characteristics of 7 instruments for detecting major depression in older adults in primary care

\begin{tabular}{|c|c|c|c|}
\hline Instrument (number of trials) & Description (cutpoints) & Sensitivity & Specificity \\
\hline Geriatric Depression Scale (9) & Primarily 15 items, yes/no format (3-5) & $79-100 \%$ & $67-80 \%$ \\
\hline Center for Epidemiologic Studies Depression scale (5) & 20 items ranking symptom frequency $(9-21)$ & $75-93 \%$ & $73-87 \%$ \\
\hline SelfCARE(D) (3) & 12 item Likert scale (5) & $77-90 \%$ & $53-98 \%$ \\
\hline Caribbean Culture-Specific Screen (2) & Number of items not reported (5-6) & $82-92 \%$ & $68-79 \%$ \\
\hline Cornell Scale for Depression in Dementia (1) & Number of items not reported (7) & $90 \%$ & $75 \%$ \\
\hline 1-question screen (from Mental Health Inventory of the SF-36) (1) & 1 item, 6 point scale (2) & $67 \%$ & $60 \%$ \\
\hline Brief Assessment Schedule Depression Cards (1) & Number of items not reported (6) & $92 \%$ & $84 \%$ \\
\hline
\end{tabular}

